\title{
Keynote Lecture
}

\section{KN10}

A decade of multiferroic research: new concepts and materials

\section{Chapon $^{1}$}

${ }^{1}$ Institut Laue Langevin, Grenoble, France

In the last 10 years, the surge of interest for multiferroics has allowed to identify several new classes of materials in which electric and magnetic degrees of freedom are highly coupled. In particular, in the so-called type II multiferroics, the onset of long range magnetic order induces ferroelectric polarization and the magnetic domains can be controlled by an electric field or conversely the direction of the polarization can be flopped by a magnetic field. I will review the most recent discovery in the field, and show how neutron and Xray magnetic scattering provide a very detailed understanding of the magnetoelectric coupling mechanism.

Keywords: Multiferroic, Magnetic diffraction 